\title{
Toward Biorelevant Dissolution: Application of a Biphasic Dissolution Model as a Discriminating Tool for HPMC Matrices Containing a Model BCS Class II Drug

\author{
Daniel J.Phillips ${ }^{1}$, Samuel R. Pygall2, V. Brett Cooper², and James C. Mann²,* \\ 'Department of Chemistry, University of Warwick, Coventry, Warwickshire, CV4 7AL, UK \\ ${ }^{2}$ Development Laboratories, MSD Development Laboratories, Hertford Road, Hoddesdon, Herts, EN11 9BU, UK
}

\begin{abstract}
The potential of a biphasic dissolution system to assist with the analysis of controlled-release (CR), Biopharmaceutics Classification System (BCS) Class II pharmaceutical products has been investigated. Use of a biphasic dissolution medium (aqueous/octanol) provided sink conditions and afforded complete dissolution of nifedipine formulated in a CR matrix tablet while maintaining the dosage form in an aqueous environment. This was not possible in a monophasic (aqueousonly) dissolution medium. Consequently, the biphasic model allowed the discrimination of three formulations containing different HPMC loadings while the monophasic medium did not. The performance of the formulations in conventional dissolution media incorporating the inorganic salt dibasic sodium phosphate, the surfactant sodium dodecyl sulfate (SDS), and ethanol as solubility modifiers was assessed. The addition of the salt and surfactant failed to produce complete discrimination in a predictive manner. The use of a hydroalcoholic medium comprising water and ethanol enabled the statistical discrimination of the three formulations but not as effectively as the biphasic medium.
\end{abstract}

\section{INTRODUCTION}

ontrolled-release (CR) matrix systems are designed to continuously deliver and maintain a drug concentration at a desired level in the body (1). Advantages of such systems include the maintenance of plasma drug concentrations in a therapeutically desired range (2), a reduction in toxic side effects (3), improved patience compliance (4), and a reduction in the required administration frequency (5). Typically the basis for such matrices are cellulose ether polymers such as hydroxypropyl methylcellulose (HPMC), which form a pseudogel layer on the surface of the tablet when exposed to water. Drug release from the system can then occur by two mechanisms: erosion of the gel layer (poorly water-soluble drugs) and dissolution and diffusion through the hydrated gel layer (soluble drugs) (6-10).

When developing a CR drug, it is common practice to design multiple formulations with different release profiles. While it is hoped that one of these designs will provide the desired pharmacokinetic (PK) profile when tested in vivo, further iterations of the formulations may be required. Reliable in vitro dissolution techniques should provide information on the stability of the product, enable accurate quality control testing, and in many cases, establish in vitro-in vivo correlations (IVIVCs) (11). Of these, an IVIVC is highly advantageous as it allows drug companies to adjust formulations without the need for further in vivo testing (12). However, the development of such biorelevant dissolution methods for CR formulations can be problematic. This is typically the case with

${ }^{*}$ Corresponding author.
Biopharmaceutics Classification System (BCS) Class II drugs, where adequate dissolution in aqueous solutions at a physiological pH cannot necessarily be achieved (13). Since many aqueous media fail to provide sink conditions for low-solubility compounds, dissolution method development can be challenging with insufficient drug release and slow release rates, two commonly encountered problems (14). Methodologies that eliminate this sink hindrance are therefore desirable to assist with formulation development.

One way of providing sink conditions is through the use of larger volumes of aqueous dissolution media, either directly (15-17) or via alternative dissolution systems such as the flow-through apparatus (USP Apparatus 4) in the open-loop configuration $(18,19)$. The former method, however, has the practical implications and expense associated with the handling of a large volume of media, while the later can consume vast volumes of media when testing CR formulations.

One of the most common and well-documented ways of providing adequate sink is through the addition of surfactants to the dissolution media. This is especially useful for the dissolution of immediate-release (IR) products (20-22) where the improved release rate has been rationalized by the ability of the surfactant to increase the hydrophilicity of the dosage form (23), act as a wetting agent (24), and increase micellar solubilization (25). However, there is substantial evidence to suggest the existence of an interaction between surfactants and cellulose ethers present in CR matrix systems. Nilsson (26) demonstrated that the addition of sodium dodecyl sulfate (SDS) to HPMC-containing solutions increased the solution 
cloud points, which was further related to an increase in polymer solubility. Alternatively, Saito (27) and Daly et al. (28) demonstrated that the addition of SDS to HPMCbased matrices increased the solution viscosity and resulted in an increased duration of drug release. Alli et al. (29) attributed this to a two-step process, namely an iondipole interaction between the polymer and surfactant, followed by hydrophobic bonding between hydrocarbon chains in surfactants associated with different polymer chains. They suggested that these chain extensions and subsequent higher molecular weights resulted in an increased viscosity. Since these interactions can modify the mechanism of release from the matrix and hence lead to a release profile that is no longer relevant in vivo, the addition of surfactants to CR formulations containing poorly water-soluble drugs requires careful consideration.

Alternative methods for improving the solubility of poorly soluble drugs are available, including the addition of inorganic salts to change the ionic strength of a given medium (30). Equally, hydrophobic cosolvents are useful at improving solubility though they provide no physiological relevance (31). Such solvents also carry the risk of an increase in dissolution variability as a result of an interaction between some of the tablet components and the medium cosolvents (32). This could be especially problematic if the cosolvent interacts with the polymer matrix.

Given the potential limitations of the previously discussed methods, an alternative method that affords complete dissolution in a realistic aqueous environment, while not interfering with the polymer matrix, is highly desirable. One such option is that of a biphasic dissolution system. The basis for such a model was initially suggested by Levy (33), who proposed that the presence of an upper organic phase within the dissolution medium could be used to act as a reservoir for the dissolved drug. Briefly, the model utilizes a dissolution medium consisting of immiscible aqueous and organic solvents from which the organic solvent, by virtue of its physical properties, will separate. Dissolution of the drug in the aqueous layer is followed by a partitioning-transfer step, thus exploiting the lipophilicty $(\log P$ ) of the compound. After partitioning, the aqueous layer dissolves more of the drug and thus the dissolution-partition cycle can continue. In this manner, the aqueous layer does not saturate, sink conditions are maintained, and the experiment will yield, in theory, complete dissolution.

There are several examples of the use of a biphasic dissolution system as a means of maintaining sink conditions, some of which look at CR formulations of poorly soluble drugs (34-41). However, there does not appear to be any example illustrating the use of a biphasic dissolution model to discriminate between CR formulations designed to release the API with different rates based on the matrix polymer content. This study ultimately aimed to illustrate the potential of a biphasic dissolution system as a simple, easy-to-implement tool utilizing the most commonly available USP 2 dissolution apparatus that could be used reproducibly as a means of maintaining sink conditions. It was hoped the model would be sensitive enough to detect changes in the release rates of $C R$ formulations containing different HPMC (polymer) loadings in a manner not possible with traditional aqueous media. To assess the relative utility of the technique, the results produced were then compared with those generated in dissolution media incorporating inorganic salts, surfactants, and cosolvents as solubility modifiers.

\section{MATERIALS AND METHODS \\ Materials}

Nifedipine, octanol, dibasic sodium phosphate, SDS, citric acid, orthophosphoric acid, ethanol, acetic acid, and acetonitrile were all obtained from Sigma Aldrich Company Ltd. (Poole, Dorset, UK). All materials were of analytical grade and used without further purification. Deionized water was generated in house. Methylcellulose (MC, Premium Methocel A4C) and hydroxypropyl methylcellulose (HPMC, Premium Methocel K100 LV) were obtained from Colorcon (Dartford, Kent, UK), microcrystalline cellulose (MCC) (Avicel PH 102) from FMC Biopolymer (Philadelphia, PA, USA), lactose monohydrate (Fast Flo 316) from Foremost Co. (Baraboo, WI, USA), and magnesium stearate (Type 2255) from Mallinckrodt Specialty Co (St Louis, MO, USA).

\section{Methods \\ Nifedipine Solubility Determination}

The solubility of nifedipine in water and octanol was determined by adding an excess of drug $(15 \mathrm{mg})$ to solvent $(1 \mathrm{~mL}$ ) in a $1.5-\mathrm{mL}$ Eppendorf tube (Eppendorf UK Ltd., Cambridge, Cambridgeshire, UK). Tubes were shaken at $37^{\circ} \mathrm{C}$ using a thermomixer (Eppendorf UK Ltd., Cambridge, Cambridgeshire, UK), and samples were prepared in duplicate at $0.5,1$, and $24 \mathrm{~h}$. Samples were centrifuged (Eppendorf UK Ltd., Cambridge, Cambridgeshire, UK) at $14000 \mathrm{rpm}$ for $10 \mathrm{~min}$. An aliquot of the supernatant was transferred to an amber vial (Agilent Technologies Inc., Santa Clara, CA, USA), and analysis was performed by the HPLC method specified below.

\section{HPLC Analysis}

All samples were analyzed by an isocratic, reversed-phase HPLC method using an Agilent 1100 chromatographic system fitted with a fixed-wavelength UV detector (Agilent Technologies Inc., Santa Clara, CA, USA). The analytical column was a $50 \times 4.6 \mathrm{~mm} \mathrm{C18}$ Onyx Monolith (Phenomenex, Inc., Macclesfield, Cheshire, UK). A mobile phase consisting of $0.1 \%$ orthophosphoric acid/acetonitrile (68:32) at a flow rate of $3.0 \mathrm{~mL} / \mathrm{min}$ was employed. The column oven temperature was maintained at $40{ }^{\circ} \mathrm{C}$, the UV detection wavelength was fixed at $238 \mathrm{~nm}$, and the chromatographic run time was $2.00 \mathrm{~min}$. The HPLC was calibrated with nifedipine standards prepared using a diluent comprising acetonitrile/deionized (50:50) water. 


\section{Nifedipine CR Formulation Preparation}

Nifedipine, HPMC, MCC, and lactose monohydrate were blended in a Turbula T2C mixer (Willy Bachofen UK, Welwyn Garden City, Hertfordshire, UK) for 10 min at a speed of $42 \mathrm{rpm}$. Magnesium stearate was sieved using a 250- $\mu \mathrm{m}$ stainless steel laboratory test sieve and added to the formulation, which was blended in the Turbula T2C mixer for an additional minute. Tablets were produced by direct compression, using a Lloyd LR50K Plus Universal Materials Testing Machine (Lloyd, Southampton, Hampshire, UK) at a constant compression force of $10 \mathrm{kN}$ and a rate of $10 \mathrm{~mm} / \mathrm{min}$ to provide tablets with a 200-mg image size.

\section{In Vitro Dissolution Studies \\ Dissolution of Nifedipine API (Proof of Concept)}

Nifedipine (6 mg) was weighed into a glass vial, $0.5 \%$ $\mathrm{MC}$ in water solution ( $3 \mathrm{~mL}$ ) was added, and the suspension was stirred for $30 \mathrm{~min}$. Nifedipine is a photosensitive compound (42-44), hence the vials were covered in foil to minimize light exposure. Dissolution studies were performed using USP Apparatus 2 in a Distek 6100 dissolution bath (Distek Inc., North Brunswick, New Jersey, USA). Testing was performed in triplicate $(n=3)$ in both monophasic (600 $\mathrm{mL}$ deionized water) and biphasic $(600 \mathrm{~mL}$ deionized water and $250 \mathrm{~mL}$ octanol) dissolution media. The aqueous layer was added to each vessel, and the bath was heated to temperature. The nifedipine suspension was added, and the glass vial was washed with $20 \mathrm{~mL}$ of dissolution media to ensure complete transfer of suspension to the vessel. Octanol was added last to three of the vessels. Both media were kept at a constant temperature of $37 \pm 0.5^{\circ} \mathrm{C}$ and agitated at a rotation speed of $50 \mathrm{rpm}$.

Dissolution of Nifedipine CR Matrix Tablets

Dissolution studies were performed using USP Apparatus 2 in a Distek 6100 dissolution bath equipped with an Evolution 4300 Autosampler (Distek Inc., New Jersey, USA). Testing was performed in triplicate $(n=3)$ for the three nifedipine formulations in both monophasic (600 mL water) and biphasic ( $600 \mathrm{~mL}$ water, $350 \mathrm{~mL}$ octanol) dissolution media and in duplicate $(n=2)$ in the conventional aqueous $(900 \mathrm{~mL}$ ) medium. The medium was kept at a constant temperature of $37 \pm 0.5^{\circ} \mathrm{C}$ and was agitated at a paddle speed of either $100 \mathrm{rpm}$ (mono/biphasic media) or $50 \mathrm{rpm}$ (conventional aqueous medium). A $\mu$ Diss Profiler (pION Inc., Woburn, MA, USA) was used to monitor the organic layer. The UV probes were fitted with a tip of $5-\mathrm{mm}$ path length and held in the organic layer with clamps. Tablets were placed in 5-coil Japanese Pharmacopeia Basket Sinkers (Quality Lab Accessories LLC, Bridgewater, NJ, USA) to restrict tablet buoyancy. Figure 1 shows the experimental setup. The dissolution bath was encased in a black plastic bag, and the lights directly above the dissolution bath were switched off at all times to minimize light exposure. All sample preparation was conducted in a room with sodium lighting, and samples were placed in amber HPLC vials before and during analysis.

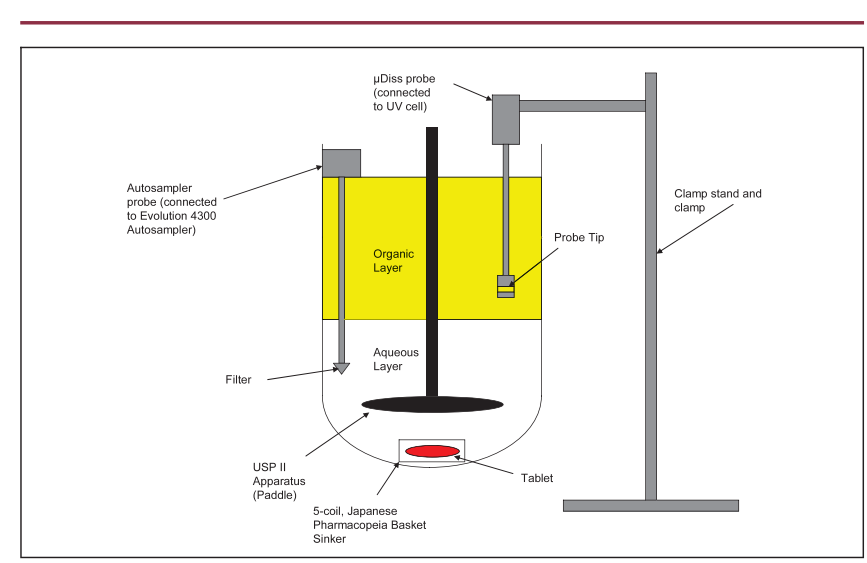

Figure 1. Biphasic dissolution experimental setup.

Dissolution Sampling and Analysis

Dissolution of Nifedipine API (Proof of Concept)

Aqueous and organic samples $(1 \mathrm{~mL})$ were taken manually at 10,20, and 30 min and 1,2, and $17 \mathrm{~h}$ using a syringe and transferred to amber HPLC vials. Each sample was centrifuged at $14000 \mathrm{rpm}$ for $5 \mathrm{~min}$. The supernatant was transferred to an amber HPLC vial, and all samples were analyzed with the HPLC method stated previously. Dissolution of Nifedipine CR Matrix Tablets

All aqueous samples were taken using the autosampler and transferred to amber HPLC vials. Samples $(1 \mathrm{~mL})$ were taken at regular intervals up to and including $20 \mathrm{~h}$ and analyzed by the HPLC method specified previously. For experiments using monophasic and biphasic media, sample time-points were $10,20,30$, and $45 \mathrm{~min}$ and $1,1.5$, $2,3,4,5,6,8,10,12,14,16,18$, and $20 \mathrm{~h}$ to maximize the number of data points obtained. With conventional aqueous media, samples were taken in accordance with USP convention for CR dosage forms, namely 0.5, 1, 2, 3, 4, $6,8,10,12,16$, and $20 \mathrm{~h}$. Analysis of the organic layer was performed using the $\mu$ Diss Profiler. The instrument was calibrated against a standard (nifedipine in octanol) of known concentration. Further standards were prepared by dilution with octanol and analyzed to produce a calibration curve. The $100 \%$ transmittance and dark spectra were recorded before each experiment, and the instrument was recalibrated monthly to verify lamp performance consistency. The software was programmed to record measurements of the organic layer in real time, which for the purpose of this work, coincided with aqueous sampling time-points. The UV detection and baseline correction wavelengths were set at $254 \mathrm{~nm}$ and $280 \mathrm{~nm}$, respectively. Dissolution profiles for biphasic media are presented as the sum of drug dissolved in aqueous and organic layers.

\section{Statistical Analysis}

A statistical tool used to assist with the interpretation and understanding of the dissolution data is the similarity factor, $f_{2}$. Initially proposed by Moore and Flanner (45), $f_{2}$ is 
defined by the FDA as a logarithmic reciprocal square-root transformation of one plus the mean squared (the average sum of squares) differences of drug percent dissolved between the test and the reference products:

$$
f_{2}=50 \cdot \log \left\{\left[1+\frac{1}{n} \sum_{j=1}^{n}\left(R_{j}-T_{j}\right)^{2}\right]^{-0.5} \times 100\right\}
$$

where $f_{2}$ is the similarity factor, $n$ is the number of observations, $R_{j}$ and $T_{j}$ are the percent of drug dissolved from reference and test formulations, respectively, at each time point $j$. Given the regulatory acceptance together with its simplicity, $f_{2}$ is widely used as part of a decision criterion to establish similarity of two formulations (46). Two dissolution profiles are considered similar if $f_{2}$ is between 50 and 100 (47). Given the sensitivity of the $f_{2}$ calculation to the number of dissolution points used, the FDA suggests only one measurement beyond $85 \%$ dissolution should be included (48). In this investigation, $f_{2}$ was used to compare formulations designed to reach $85 \%$ dissolution at different times. Therefore, for any two profiles under comparison, the sample inclusion cutoff became the point at which the first curve exhibited a plateau. In some cases, this included more than one point after $85 \%$; however, for our purpose this was deemed a suitably robust way of assessing the relative similarity. For profiles exhibiting low dissolution percentages (i.e., where $85 \%$ was not attained), the whole profile was used.

\section{RESULTS AND DISCUSSION Proof of Concept}

In this study, the calcium-channel-blocking agent nifedipine was chosen as a model drug because of its physical properties. Classified as a BCS Class II drug (49), it has a $\mathrm{p} K_{\mathrm{a}} \approx 1.0(50)$ indicating that as a very weak base, it would remain un-ionized in the dissolution media used throughout the study. The drug also has a low aqueous solubility that remains approximately constant across the $\mathrm{pH}$ range $2-13(\approx 0.6 \mu \mathrm{g} / \mathrm{mL})(51,52)$. This value concurs with the experimentally determined solubility of nifedipine in water (Table 1, value at $24 \mathrm{~h}$ taken as the equilibrium solubility), which illustrates that sink conditions may not be possible in most aqueous dissolution media.

The oral absorption of small molecules is largely determined by their ability to cross the GI tract, a process mediated by passive diffusion through the lipid membrane (53). Octanol, like membrane lipids, has a hydrophobic, long alkyl chain and a polar hydroxyl group (54). The ratio of un-ionized drug concentration in the organic and aqueous phases at equilibrium is the partition coefficient $(\log P)$. In accordance with this, water and octanol were selected as the aqueous and organic solvents used in the biphasic dissolution medium throughout this study, respectively. It should be noted that previous studies have reported biphasic media comprising a variety of alternative aqueous (SIF, $\mathrm{HCl}, \mathrm{SDS}$, and sodium phosphate-buffered water) and organic (chloroform, ethyl
Table 1. Solubility of Nifedipine in Water and Octanol at 0.5, 1 , and $24 \mathrm{~h}$ (Mean values shown, $n=2$ )

\begin{tabular}{lcc}
\hline Solvent & Time (h) & Solubility $(\mathbf{m g} / \mathbf{m L})$ \\
\hline Octanol & 0.5 & 2.3 \\
\hline 1.0 & 2.4 \\
\hline Water & 24.0 & 9.6 \\
\hline & 0.5 & $5.6 \times 10^{-3}$ \\
\hline & 1.0 & $5.7 \times 10^{-3}$ \\
\hline & 24.0 & $7.5 \times 10^{-3}$ \\
\hline
\end{tabular}

acetate, cyclohexene/octanol, and nonanol/octanol) solvents (34-41).

$\log P$ is a routinely measured parameter used to predict drug absorption (55). It has been suggested the $\log P$ values between 0 and 3 constitute an optimal window for drug absorption. Values that are too high $(>6)$ or too low $(<-3)$ have been associated with poor transport characteristics. High $\log P$ values correspond to compounds with poor aqueous solubility. Compounds with low $\log P$ values have a limited ability to penetrate membrane barriers (56). The relatively lipophilic nature of nifedipine is reflected by a $\log P$ value of 2.86 (57) which, coupled with high octanol solubility (approximately $10 \mathrm{mg} / \mathrm{mL}$, Table 1 ), suggested it as a good model drug because (1) it would partition successfully and (2) the biphasic system would provide sink conditions for dissolution.

To verify this, the dissolution of nifedipine API in both monophasic and biphasic media was performed. The paddle speed was set at $50 \mathrm{rpm}$ in accordance with the USP dissolution method for Nifedipine Extended Release Tablets (test 2)and FDA recommendations $(58,59)$, and arbitrary aqueous and organic volumes were selected such that sufficient aqueous dissolution could occur while maintaining organic sink conditions. The results confirmed the biphasic medium enabled greater drug solubility, in this case allowing a two-fold increase in percent dissolved over the course of the experiment compared with the monophasic medium. It also demonstrated that the drug, once dissolved in the aqueous solvent, partitioned rapidly into the organic layer.

\section{Dissolution Method Development and Analysis of Nifedipine CR Matrix Tablets in Monophasic and Biphasic Media}

The dissolution of three nifedipine CR matrix formulations in both monophasic and biphasic media was then assessed. As shown in Table 2, slow, medium, and fast CR formulations consisting of nifedipine $(10 \% \mathrm{w} / \mathrm{w}), \mathrm{HPMC}$ K100LV (10, 20, or 40\% w/w), MCC, lactose monohydrate, and magnesium stearate were prepared. Their respective release rates were engineered by altering the HPMC loading in each blend. Increasing the polymer loading will increase the tortuosity of the matrix, and the release rate of the poorly soluble nifedipine will be slower. 
Table 2. Composition of Nifedipine CR Formulations Analyzed

\begin{tabular}{|c|c|c|c|c|c|c|}
\hline & \multicolumn{2}{|c|}{ Slow-Release Rate } & \multicolumn{2}{|c|}{ Medium-Release Rate } & \multicolumn{2}{|c|}{ Fast-Release Rate } \\
\hline Component & $\begin{array}{l}\text { Mass per tablet } \\
(\mathrm{mg})\end{array}$ & $\% w / w$ & $\begin{array}{l}\text { Mass per tablet } \\
\text { (mg) }\end{array}$ & $\% \mathbf{w} / \mathbf{w}$ & $\begin{array}{l}\text { Mass per tablet } \\
\text { (mg) }\end{array}$ & $\%$ w/w \\
\hline Nifedipine & 20 & 10 & 20 & 10 & 20 & 10 \\
\hline MCC (diluent/filler) & 30 & 15 & 30 & 15 & 30 & 15 \\
\hline $\begin{array}{l}\text { HPMC K100LV (functional } \\
\text { excipient) }\end{array}$ & 80 & 40 & 40 & 20 & 20 & 10 \\
\hline Lactose Monohydrate (filler) & 68 & 34 & 108 & 54 & 128 & 64 \\
\hline Magnesium Stearate (lubricant) & 2 & 1 & 2 & 1 & 2 & 1 \\
\hline Total & 200 & 100 & 200 & 100 & 200 & 100 \\
\hline
\end{tabular}

The dissolution parameters were optimized following preliminary work with the medium-release tablets. Both layers were monitored throughout the entire experiment using a $\mu$ Diss profiler (organic layer) and an autosampler (aqueous layer). An optimum paddle speed of $100 \mathrm{rpm}$ was selected to ensure instant homogenization of drug in the organic layer following partitioning. The extent of solvent mixing before the experiment start was also assessed and determined to have no effect. The biphasic medium was $600 \mathrm{~mL}$ aqueous and $350 \mathrm{~mL}$ organic. The aqueous volume was selected to enable facile sampling by the autosampler probes, while a volume of $350 \mathrm{~mL}$ was sufficient to immerse the fiber-optic probe in the organic layer. The dissolution is initiated in the aqueous solvent while the organic layer acts as a drug reservoir, and can hence be considered as an external phase. Consequently, the monophasic (aqueous only) medium consisted of 600 $\mathrm{mL}$ water to mirror the aqueous content of the biphasic medium.

Dissolution in monophasic media (Figure 2) failed to provide sink conditions. All formulation dissolution profiles reached a plateau at approximately $28 \%$ (solubility

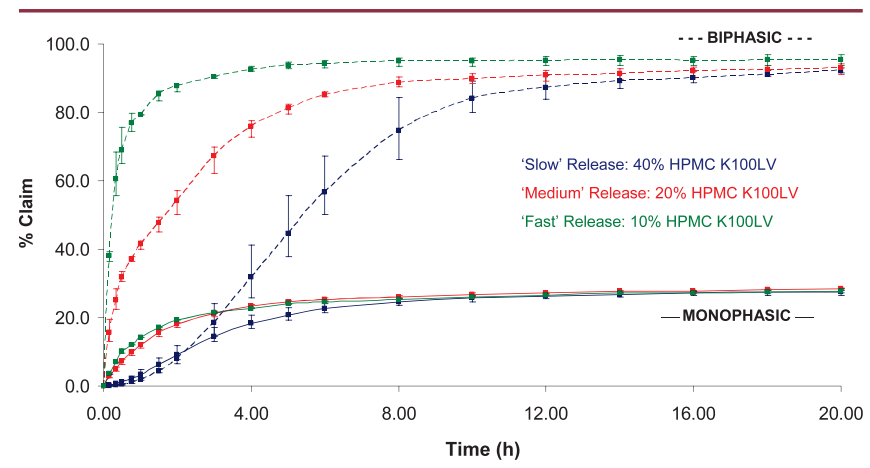

Figure 2. Dissolution of nifedipine slow-, medium-, and fast-release rate $C R$ tablets in monophasic (600 mL aqueous, solid lines) and biphasic (600 mL aqueous, $350 \mathrm{~mL}$ organic, dashed lines) dissolution media. Data obtained using USP Apparatus 2 with a rotation speed of $100 \mathrm{rpm}$. Bath held at

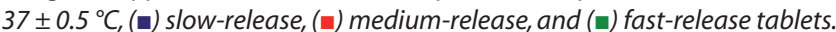
Mean values $(n=3)$ are shown; error bars represent maximum and minimum values. of $0.009 \mathrm{mg} / \mathrm{mL}$ in reasonable agreement with the experimentally determined water solubility of nifedipine) with the profiles appearing identical from $8 \mathrm{~h}$ onward. In the initial $6 \mathrm{~h}$, the slow-release profile was different from the medium- and fast-release tablets. The largest difference can be seen when comparing the slow- and fast-release tablets at $1 \mathrm{~h}$, where the percent claim differs by $11 \%$. The medium- and fast-release tablets seem to have very similar release rates throughout with the largest difference in percent dissolved between the two being $2.3 \%$ at $1 \mathrm{~h}$. Calculations of $f_{2}$ for the monophasic media are shown in Table 3. By comparing the three profiles against one another, the data indicate that all profiles are similar. It would not be possible to discriminate among the formulations in water.

Dissolution was then performed in the biphasic medium (Figure 2) where, as hoped, sink conditions were maintained with the organic layer providing a reservoir into which the lipophilic drug can partition following aqueous dissolution. The true release rate of the three formulations was determined by providing conditions for complete drug dissolution. As a result, clear discrimination between all formulations was possible and confirmed by $f_{2}$ calculations (Table 4).

\section{Model Verification: Dissolution of Nifedipine Formulations in Conventional Aqueous Media}

The capability of the biphasic dissolution model to discriminate among nifedipine $\mathrm{CR}$ formulations containing different polymer loadings has been demonstrated. The ability of aqueous media with different ionic strengths and

Table 3. Assessment of the Similarity of Profiles Obtained from the Dissolution of Nifedipine CR Tablets with Different Release Rates in Monophasic Dissolution Media: $f_{2}$ Calculations

\begin{tabular}{lccc}
\hline $\begin{array}{l}\text { Calculation } \\
\text { Number }\end{array}$ & $\begin{array}{c}\text { Tablet Release } \\
\text { Rate } \mathbf{1}\end{array}$ & $\begin{array}{c}\text { Tablet Release } \\
\text { Rate } \mathbf{2}\end{array}$ & $\boldsymbol{f}_{\mathbf{2}}$ Value \\
\hline 1 & Slow & Medium & 64.8 \\
\hline 2 & Slow & Fast & 61.3 \\
\hline 3 & Medium & Fast & 89.4 \\
\hline
\end{tabular}

Dissolution Technologies | FEBRUARY 2012 
Table 4. Assessment of the Similarity of Profiles Obtained from the Dissolution of Nifedipine CR Tablets with Different Release Rates in Biphasic Dissolution Media: $f_{2}$ Calculations

\begin{tabular}{lccc}
\hline $\begin{array}{l}\text { Calculation } \\
\text { Number }\end{array}$ & $\begin{array}{c}\text { Tablet Release } \\
\text { Rate } \mathbf{1}\end{array}$ & $\begin{array}{c}\text { Tablet Release } \\
\text { Rate } \mathbf{2}\end{array}$ & $\mathbf{f}_{\mathbf{2} \text { Value }}$ \\
\hline 1 & Slow & Medium & 23.1 \\
\hline 2 & Slow & Fast & 9.2 \\
\hline 3 & Medium & Fast & 26.3 \\
\hline
\end{tabular}

surfactant concentrations and a hydroalcoholic medium to provide the same degree of discrimination was assessed.

Given their widespread use in dissolution media, dibasic sodium phosphate and SDS were chosen as the salt and surfactant, respectively. The salt was added to the media at minimum and maximum concentrations of $10 \mathrm{mM}$ and $150 \mathrm{mM}$, respectively. Additional media were prepared at the same concentrations together with $5 \%$ SDS. The final $\mathrm{pH}$ of the dissolution media was fixed at $\mathrm{pH} 6.8$ given its physiological relevance (60), and a volume of $900 \mathrm{~mL}$ was used. Figure $3 A, B$ shows the results obtained from the buffers containing only the salt. Figure $4 A, B$ shows the results obtained from the buffers containing both the salt and $5 \%$ surfactant. Table 5 summarizes the $f_{2}$ values for these experiments.

(A)

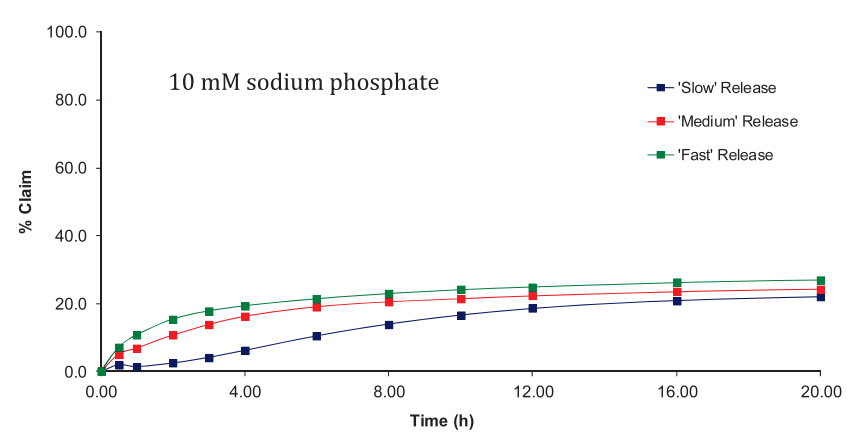

(B)

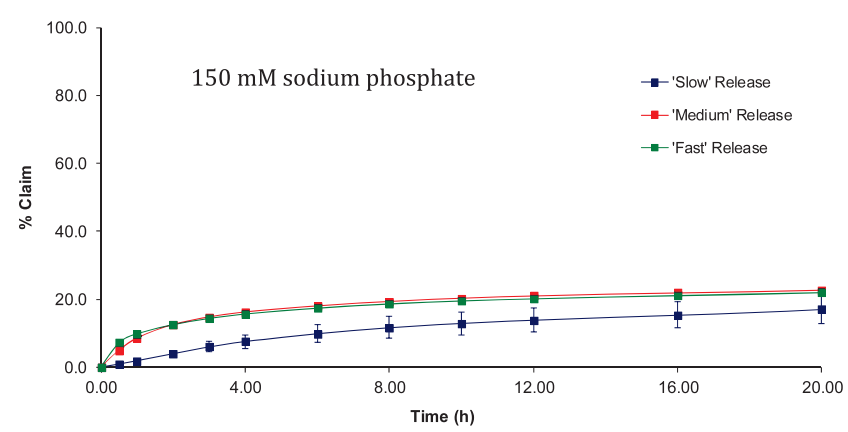

Figure 3. Dissolution of slow-, medium-, and fast-release rate nifedipine $C R$ tablets in (A) $10 \mathrm{mM}$ buffer and (B) $150 \mathrm{mM}$ buffer, pH 6.8. Data obtained using USP Apparatus 2 in $900 \mathrm{~mL}$ dissolution media at $37 \pm 0.5^{\circ} \mathrm{C}$. Rotation

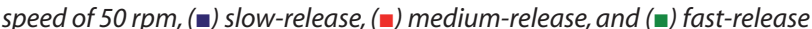
tablets. Mean values $(n=2)$ are shown; error bars represent maximum and minimum values.

Dissolution Technologies | FEBRUARY 2012
(A)

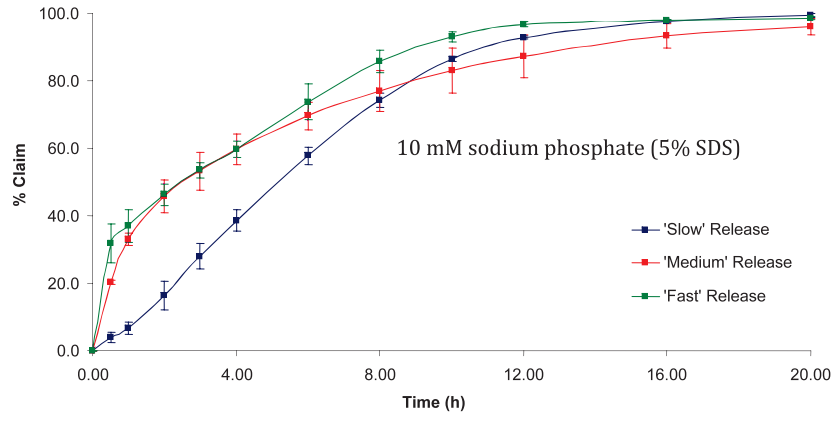

(B)

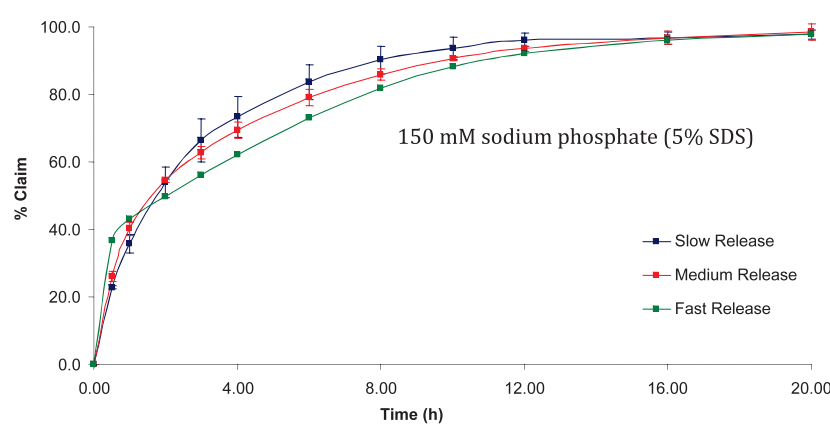

Figure 4. Dissolution of slow-, medium-, and fast-release rate nifedipine $C R$ tablets in (A) $10 \mathrm{mM}$ buffer (5\% SDS) and (B) $150 \mathrm{mM}$ buffer (5\% SDS), pH 6.8. Data obtained using USP Apparatus 2 in $900 \mathrm{~mL}$ dissolution media at $37 \pm 0.5^{\circ} \mathrm{C}$. Rotation speed of $50 \mathrm{rpm}$, (घ) slow-release, (घ) medium-release, and $(\square)$ fast-release tablets. Mean values $(n=2)$ are shown; error bars represent maximum and minimum values.

Dissolution in $10 \mathrm{mM}$ buffer (Figure 3A) failed to provide sink conditions. The solubility at $20 \mathrm{~h}$ was $9 \mu \mathrm{g} / \mathrm{mL}$, and an $f_{2}$ calculation indicates the similarity of the three formulations in this medium (Table 5). The use of a $150 \mathrm{mM}$ buffer (Figure 3B) produced results with a greater degree of similarity. The dissolution rate of the drug increased, with the medium- and fast-formulations releasing at equivalent rates. It is well established that increasing the ionic strength in a polymer solution reduces polymer solubility. This can be attributed to the salting out of the polymer by the ions present in the media. Drug release is more rapid as the polymer chains lose water of hydration due to the additional competition provided by the ions.

Consequently, the polymer is less able to form a protective pseudogel layer $(6,61)$. The results observed above are consistent with work reported elsewhere. Johnson et al. (62) investigated the effect of solutions with varying ionic strengths on the matrix integrity and drug release from hydroxypropyl cellulose (HPC) matrix compacts. They found that the dissolution rate of the compacts increased with increasing ionic strength. A similar observation was made by Mitchell et al. (63) for the dissolution of propranolol hydrochloride from HPMC matrices.

The addition of SDS afforded complete dissolution because of an increase in drug solubility. Dissolution in $10 \mathrm{mM}$ buffer 


\begin{tabular}{|c|c|c|c|c|c|}
\hline $\begin{array}{l}\text { Calculation } \\
\text { Number }\end{array}$ & $\begin{array}{c}\text { lonic } \\
\text { Strength } \\
(\mathrm{Mm})\end{array}$ & $\begin{array}{c}\text { SDS } \\
\text { concentra- } \\
\text { tion (\%) }\end{array}$ & $\begin{array}{c}\text { Tablet } \\
\text { Release } \\
\text { Rate } 1\end{array}$ & $\begin{array}{c}\text { Tablet } \\
\text { Release } \\
\text { Rate } 2\end{array}$ & $f_{2}$ Value \\
\hline 1 & 10 & 0 & Slow & Medium & 59.0 \\
\hline 2 & 10 & 0 & Slow & Fast & 51.0 \\
\hline 3 & 10 & 0 & Medium & Fast & 74.2 \\
\hline 4 & 150 & 0 & Slow & Medium & 56.5 \\
\hline 5 & 150 & 0 & Slow & Fast & 57.2 \\
\hline 6 & 150 & 0 & Medium & Fast & 92.8 \\
\hline 7 & 10 & 5 & Slow & Medium & 38.6 \\
\hline 8 & 10 & 5 & Slow & Fast & 34.8 \\
\hline 9 & 10 & 5 & Medium & Fast & 57.2 \\
\hline 10 & 150 & 5 & Slow & Medium & 73.5 \\
\hline 11 & 150 & 5 & Slow & Fast & 55.2 \\
\hline 12 & 150 & 5 & Medium & Fast & 58.4 \\
\hline
\end{tabular}

with $5 \%$ SDS (Figure 4A) enabled discrimination of the slow formulation while the other two remained statistically similar to each other. Dissolution in $150 \mathrm{mM}$ buffer with 5\% SDS (Figure 4B) did not allow any discrimination and afforded a faster dissolution rate than $10 \mathrm{mM}$.

Dissolution was no longer fully predictive with the addition of surfactant. In 10 mM buffer with 5\% SDS, the medium and fast formulations had identical release rates between 1 and $4 \mathrm{~h}$. After this, the fast formulation apparently released slower than the medium formulation, before arguably demonstrating the slowest release rate of all three formulations from $12 \mathrm{~h}$ onward. In a $150 \mathrm{mM}$ buffer with $5 \%$ SDS, the profiles only trend in the expected manner for the initial 30 min time-point. For the remaining dissolution, the blend expected to release the fastest was instead the slowest, while the other two formulations are within error throughout. This can be rationalized by considering the combined effects of the salt and the surfactant on the HPMC matrix.

The addition of inorganic ions disrupts the swelling ability of HPMC and produces a faster rate of drug release. In the above experiments, the addition of the salt did not increase the drug solubility. As specified above, SDS also interacts with HPMC and increases the release rate. The addition of SDS to the dissolution media enabled all drug to dissolve. If the combined effect of salt and SDS affects the swelling capacity of the polymer to a larger extent than the salt alone, it is plausible that the HPMC can no longer control the drug release in the usual manner. Consequently, dissolution of the three formulations may not correlate with the respective polymer loadings, while the profiles produced may be both unpredictable and variable.

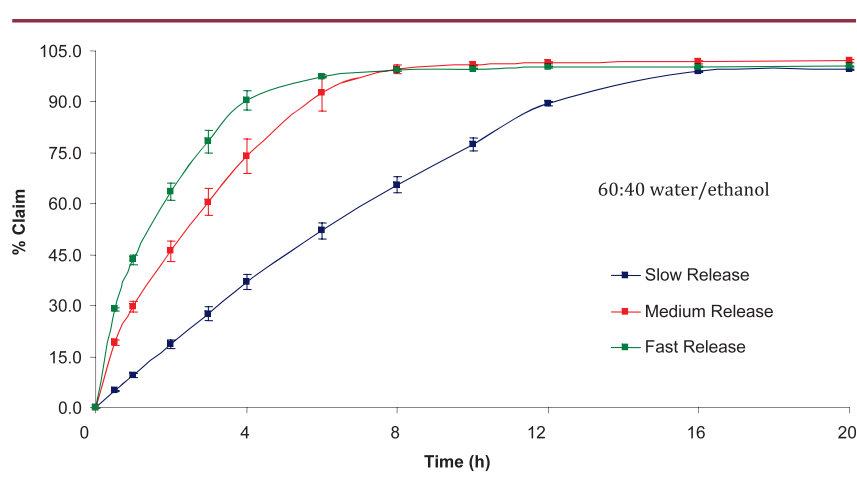

Figure 5. Dissolution of slow-, medium-, and fast-release rate nifedipine $C R$ tablets in 60:40 water/ethanol. Data obtained using USP Apparatus 2 in 900 $\mathrm{mL}$ dissolution media at $37 \pm 0.5^{\circ} \mathrm{C}$. Rotation speed of $50 \mathrm{rpm},(\mathbf{\square})$ slowrelease, ( $\square$ ) medium-release, and ( $\square$ ) fast-release tablets. Mean values $(n=2)$ are shown; error bars represent maximum and minimum values.

Ethanol is a common choice of cosolvent. In 2005, the CR dosage form of the opioid analgesic Palladone was discovered to break down when ingested with alcohol. The peak plasma concentration of the active ingredient, hydromorphone, increased to lethal levels, and the drug was consequently withdrawn from the market (64). Testing with ethanol is commonly used to assess the propensity for dose-dumping to occur with other drugs. A common choice of ethanol concentration is $40 \%$, the concentration found in many spirits (65-67).

Calpena et al. (68) determined that the solubility of nifedipine in a 50:50 ethanol/water solution is $6.5 \mathrm{mg} / \mathrm{mL}$. It was hence believed that sink conditions would be maintained in a $40 \%$ ethanolic solution. This experiment was performed to assess the ability of a dissolution medium of $60: 40$ water/ethanol to discriminate between the nifedipine CR formulations. The data are presented in Figure 5.

The hydroalcoholic medium enabled all formulations to be discriminated in a manner only seen previously using the biphasic medium (Table 6 lists the $f_{2}$ calculations for these data). While the slow formulation was statistically different in some of the other media investigated, the hydroalcoholic medium was the only one to provide an $f_{2}$ value less than 50 when comparing the medium and fast formulations. Moreover, the data are fully predictive. This discrimination can be explained by considering the ability of the ethanolic solution to maintain sink conditions because of the hydrophobic nature of the medium.

Table 6. Assessment of the Similarity of Profiles Obtained from the Dissolution of Nifedipine CR Tablets with Different Release Rates in Hydroalcoholic Dissolution Media: $f_{2}$ Calculations

\begin{tabular}{lccc}
\hline $\begin{array}{l}\text { Calculation } \\
\text { Number }\end{array}$ & $\begin{array}{c}\text { Nifedipine Tablet } \\
\text { Release Rate 1 }\end{array}$ & $\begin{array}{c}\text { Nifedipine } \\
\text { Tablet Release } \\
\text { Rate 2 }\end{array}$ & $\mathbf{f}_{\mathbf{2}}$ Value \\
\hline 1 & Slow & Medium & 25.6 \\
\hline 2 & Slow & Fast & 18.8 \\
\hline 3 & Medium & Fast & 46.4 \\
\hline
\end{tabular}

Dissolution Technologies | FEBRUARY 2012 
The discrimination is not as powerful as that produced with the biphasic model, and some differences in the release profiles do exist. Firstly, dissolution of the slow formulation in the hydroalcoholic medium is faster initially. The plot follows near zero-order kinetics before beginning to plateau at $12 \mathrm{~h}$. By comparison, a sigmoidal profile is generated from the dissolution in a biphasic medium. Secondly, the fast formulation is slower in the hydroalcoholic compared with the biphasic media, where $80 \%$ claim is achieved in approximately $3 \mathrm{~h}$ and $1 \mathrm{~h}$, respectively. These differences could be attributed to the different dissolution environments present in the two media. Dissolution in the hydroalcoholic medium exposes the tablet to different hydrophobic interactions and a substantially different wetting environment compared with the aqueous layer of the biphasic medium. The biphasic system does provide a distinct advantage in allowing the matrix to operate in a fully aqueous environment. This might be considered more relevant than the ethanolic system to in vivo conditions.

\section{CONCLUSIONS AND FUTURE WORK}

The biphasic model has demonstrated potential as a facilitative dissolution tool in CR formulation development. An optimized water/octanol system enabled discrimination among nifedipine formulations with different polymer loadings. The same discrimination was not achieved in water or media incorporating dibasic sodium phosphate and SDS. Use of buffers containing sodium phosphate failed to remove the sink limitation. Addition of SDS, while affording sink conditions, failed to adequately predict the expected trend. This was attributed to a breakdown of the tablet matrix by the SDS. Use of a hydroalcoholic medium allowed statistical discrimination of the three nifedipine formulations as sink conditions were maintained because of the increased hydrophobicity of the dissolution medium. Discrimination was not as conclusive as with the biphasic system, however, while the relative biorelevance of hydroalcoholic media remains a concern.

Given the large number of BCS Class II drugs (50-60\%) in pharmaceutical drug pipelines (69), this work will be useful in the analysis of poorly soluble, highly permeable drugs. Other BCS Class II compounds should be studied to assess the model's potential for widespread application. It should also be emphasized that this model is useful specifically for those drugs that are sink limited in traditional dissolution media. While it is anticipated that BCS Class I compounds can be analyzed using a biphasic system, their high solubility removes the challenge associated with generating sink conditions (70). Of greater interest would be the assessment of poorly permeable drugs (BCS Classes III and IV), where the potentially limited partitioning ability of such drugs following aqueous dissolution may pose analytical issues (71). Further work to model the partitioning rate would be necessary to aid the selection of an optimum organic solvent. An extension of this study should focus on elucidating the compatibility of the biphasic model with a variety of other BCS Class II drugs and on investigating its potential compatibility with BCS Class III and IV drugs.

\section{ACKNOWLEDGMENTS}

The authors would like to acknowledge Dr. Fabio Chiti for his assistance with the design and analysis of the DoE work.

\section{REFERENCES}

1. Langer, R. Implantable controlled release systems. Pharmacol. Ther. 1983, 21 (1), 35-51.

2. Kost, J.; Langer, R. Controlled release of bioactive agents. Trends Biotechnol. 1984, 2 (2), 47-51.

3. Löbenberg, R.; Kim, J. S.; Amidon, G. L. Pharmacokinetics of an immediate release, a controlled release and a two pulse dosage form in dogs. Eur.J.Pharm. Biopharm. 2005, 60 (1), 17-23.

4. Caldwell, H.C.;Westlake, W. J.; Schriver, R. C.; Bumbier, E. E. Steady-State Lithium Blood Level Fluctuations in Man Following Administration of a Lithium Carbonate Conventional and Controlled-Release Dosage Form. J. Clin. Pharmacol. 1981, 21 (2), 106-109.

5. Tiwari, S. B.; Murthy, T. K.; Pai, M. R.; Mehta, P. R.; Chowdary, P.B. Controlled Release Formulation of Tramadol Hydrochloride Using Hydrophilic and Hydrophobic Matrix System. AAPS PharmSciTech 2003, 4 (3), E31.

6. Alderman, D. A. A Review of Cellulose Ethers in Hydrophilic Matrices for Oral Controlled-Release Dosage Forms. Int. J. Pharm. 1984, 5, 1-9.

7. Ford, J. L. Thermal Analysis of Hydroxypropylmethylcell ulose and Methylcellulose: Powders, Gels and Matrix Tablets. Int. J. Pharm. 1999, 179, 209-228.

8. Li, C. L.; Martini, L. G.; Ford, J. L.; Roberts, M. The Use of Hypromellose in Oral Drug Delivery. J. Pharm. Pharmacol. 2005, 57, 533-546.

9. Skoug, J.W.; Mikelsons, M.V.; Vigneron, C. N.; Stemm, N. L. Qualitative Evaluation of the Mechanism of Release of Matrix Sustained Release Dosage Forms by Measurement of Polymer Release. J. Control. Release 1993, 27, 227-245.

10. Sung, K. C.; Nixon, P. R.; Skoug, J.W.; Ju, T. R.; Gao, P.; Topp, E. M.; Patel, M.V. Effect of Formulation Variables on Drug and Polymer Release from HPMC-Based Matrix Tablets. Int. J. Pharm. 1996, 142, 53-60.

11. Dyas, A.M.; Shah, U. U.Dissolution and Dissolution Testing. In Encyclopedia of Pharmaceutical Technology, 3rd ed.; Swarbrick, J., Ed.; Informa Healthcare USA, Inc.: New York, 2007; Vol. 5, pp 908-928.

12. Gaynor, C.; Dunne, A.; Davis, J. A Comparison of the Prediction Accuracy of Two IVIVC Modeling Techniques. J. Pharm. Sci. 2008, 97, 3422-3432.

13. Brown, C. K.; Chokshi, H. P.; Nickerson, B.; Reed, R. A.; Rohrs, B. R.; Shah, P. A. Acceptable Analytical Practices for Dissolution Testing of Poorly Soluble Compounds. Pharm. Technol. 2004, 28, 56-65.

14. Lee, J.; Park, S-A.; Sah, H. Surfactant Effects upon Dissolution Patterns of Carbamazepine Immediate Release Tablets. Arch. Pharm. Res. 2005, 28 (1), 120-126. 
15. Katchen, B.; Symchowicz, S. Correlation of Dissolution Rate and Griseofulvin Absorption in Man.J.Pharm.Sci. 1967, 56, 1108-1111.

16. Symchowicz, S.; Katchen, B. Griseofulvin Absorption in Man after Single and Repeated Treatment and its Correlation with Dissolution Rates.J.Pharm. Sci. 1968, 57, 1383-1386.

17. Chiou, W. L.; Riegleman, S. Oral Absorption of Griseofulvin in Dogs: Increased Absorption via Solid Dispersion in Polyethylene Glycol 6000. J. Pharm. Sci. 1970, 59, 937-942.

18. Posti, J.; Speiser, P. P. Sink Conditions in the FlowThrough Cell during Dissolution. Int.J. Pharm. 1980, 5, 101-107.

19. Butler,W.C.G.; Bateman, S. R. A Flow-Through Dissolution Method for a Two-Component Drug Formulation where the Actives have Markedly Differing Solubility Properties. Int. J. Pharm. 1998, 173, 211-219.

20. Ruddy, S. B.; Matuszewska, B. K.; Grim, Y. A.; Ostovic, D.; Storey, D. E. Design and Characterization of a SurfactantEnriched Tablet Formulation for Oral Delivery of a Poorly Water-Soluble Immunosuppressive Agent. Int. J.Pharm. 1999, 182, 173-186.

21. Chen, L. R.; Wesley, J. A.; Bhattachar, S.; Ruiz, B.; Bahash, K.; Babu, S. R. Dissolution Behavior of a Poorly Water Soluble Compound in the Presence of Tween 80. Pharm. Res. 2003, 20, 797-801.

22. Qazi, S.; Peter Samuel, N. K.; Venkatachalam, T. K.; Uckun, F. M. Evaluating Dissolution Profiles of an Anti-HIV Agent using ANOVA and Non-Linear Regression Models in JMP Software. Int. J. Pharm. 2003, 252, 27-39.

23. Heng, P.W. S.; Wan, L. S. C.; Ang, T. S. H. Role of Surfactant on Drug Release from Tablets. Drug Dev. Ind. Pharm. 1990, 16, 951-962.

24. Hanaee, J.; Javadzadeh, Y.; Taftachi, S.; Farid, D.; Nokhodchi, A. The Role of Various Surfactants on the Release of Salbutamol from Suppositories. Farmaco 2004, 59 (11), 903-906.

25. Allaboun, H.; Alkhamis, K. A.; Al Jabour, N. D. Effect of Surfactant on Dissolution of Spherical Particles in Micellar Systems. Eur. J. Pharm. Biopharm. 2007, 65, 188-197.

26. Nilsson, S. Interactions between Water-Soluble Cellulose Derivatives and Surfactants. 1.The HPMC/ SDS/Water System. Macromolecules 1995, 28 (23), 7837-7844.

27. Saito, S. Binding of Surfactants by Polymers. J. Colloid Sci. 1960, 15, 282-286.

28. Daly, P. B.; Davis, S. S.; Kennerley, J.W. The Effect of Anionic Surfactants on the Release of Chlorpheniramine from a Polymer Matrix. Int. J. Pharm. 1984, 18, 201-205.

29. Alli, D.; Bolton, S.; Gaylord, N. G. Hydroxypropylmethylc ellulose-Anionic Surfactant Interactions in Aqueous Systems. J. Appl. Polym. Sci. 2003, 42, 947-956.
30. Bonferoni, M.C.; Rossi, S.; Ferrari, F.; Stavik, E.; Pena-Romero, A.; Caramella, C. Factorial Analysis of the Influence of Dissolution Medium on Drug Release From Carrageenan-Diltiazem Complexes. AAPS PharmSciTech 2000, 1, 72-79.

31. Walkling, W.D.; Natak, R. K.; Plostnieks, J.; Cressman, W. A. A Partially Organic Dissolution Medium for Griseofulvin Dosage Forms. Drug Dev. Ind. Pharm. 1979, 5, 17-27.

32. El-Massik, M. A.; Darwish, I. A.; Hassan, E. E.; ElKhordagui, L. K. Development of a Dissolution Medium for Glibenclamide. Int. J. Pharm. 1996, 140, 69-76.

33. Levy, G. Papers Presented Before the Industrial Pharmacy Section. American Pharmaceutical Association: Dallas Meeting; 1966, pp 233-252.

34. Grundy, J. S.; Anderson, K. E.; Rogers, J. A.; Foster, R.T. Studies on Dissolution Testing of the Nifedipine Gastrointestinal Therapeutic System. I. Description of a Two-Phase In Vitro Dissolution Test.J. Control. Release 1997, 48, 1-8.

35. Gibaldi, M.; Feldman, S. Establishment of Sink Conditions in Dissolution Rate Determinations. J. Pharm. Sci. 1967, 56, 1238-1242.

36. Chaudhary, R. S.; Gangwal, S. S.; Gupta, V. K.; Shah, Y. N.; Jindal, K. C.; Khanna, S. Dissolution System for Nifedipine Sustained Release Formulations. Drug Dev. Ind. Pharm. 1994, 20, 1267-1274.

37. Ngo, T.H.; Michoel, A.; Kinget, R. Dissolution Testing of Artemisinin Solid Oral Dosage Forms. Int. J. Pharm. 1996, 138, 185-190.

38. Hoa, N.T.; Kinget, R. Design and Evaluation of Two-Phase Partition-Dissolution Method and its Use in Evaluating Artemisinin Tablets. J. Pharm. Sci. 1996, 85, 1060-1063.

39. Grundy, J. S.; Anderson, K. E.; Rogers, J. A.; Foster, R.T. Studies on Dissolution Testing of the Nifedipine Gastrointestinal Therapeutic System. II. Improved In Vitro-In Vivo Correlation Using a Two-Phase Dissolution Test. J. Control. Release 1997, 48, 9-17.

40. Vangani, S.; Li, X.; Zhou, P.; Del-Barrio, M-A.; Chiu, R.; Cauchon, N.; Gao P.; Medina, C.; Jasti, B. Dissolution of Poorly Water-Soluble Drugs in Biphasic Media Using USP 4 and Fiber Optic System. Clin. Res. Regul. Aff. 2009, 26, 8-19.

41. Heigoldt, U.; Sommer, F.; Daniels, R.; Wagner, K-G. Predicting In Vivo Absorption Behavior of Oral Modified Release Dosage Forms Containing pH-Dependent Poorly Soluble Drugs using a Novel pH-Adjusted Biphasic In Vitro Dissolution Test. Eur. J. Pharm. Biopharm. 2010, 76 (1), 105-111.

42. Hamann, S. R.; McAllister, R. G. Jr. Measurement of Nifedipine in Plasma by Gas-Liquid Chromatography and Electron-Capture Detection. Clin. Chem. 1983, 29 (1), 158-160.

43. Squella, J. A.; Barnafi, E.; Perna, S.; Nuñez-Vergara, L. J. Nifedipine: Differential Pulse Polarography and Photodecomposition. Talanta 1989, 36, 363-366. 
44. Suzuki, H.; Fujiwara, S.; Kondo, S.; Sugimoto, I. Determination of Nifedipine in Human Plasma by High-Performance Liquid Chromatography with Electrochemical Detection. J. Chromatogr. B 1985, 341, 341-347.

45. Moore, J.W.; Flanner, H. H. Mathematical Comparison of Dissolution Profiles. Pharm. Tech. 1996, 20, 64-74.

46. Ocaña, J.; Frutos, G.; Sánchez, O, P. Using the Similarity Factor $f_{2}$ in Practice: A Critical Revision and Suggestions for its Standard Error Estimation. Chemometr. Intell. Lab. 2009, 99, 49-56.

47. Ma, M-C.; Lin, R-P.; Liu, J-P. Statistical Evaluations of Dissolution Similarity. Stat. Sinica 1999, 9, 1011-1027.

48. Shah, V. P.; Tsong, Y.; Sathe, P.; Williams, R. L. Dissolution Profile Comparison Using Similarity Factor, $f_{2}$. Dissolution Technol. 1999, 6 (3), 15.

49. Lindenberg, M.; Kopp, S.; Dressman, J. B. Classification of Orally Administered Drugs on the World Health Organization Model list of Essential Medicines according to the Biopharmaceutics Classification System. Eur. J. Pharm. Biopharm. 2004, 58, 265-278.

50. Uehara, A.; Hume, J. R. Interactions of Organic Calcium Channel Antagonists with Calcium Channels in Single Frog Atrial Cells. J. Gen. Physiol. 1985, 85, 621-647.

51. Ali, S. L. Nifedipine. In Analytical Profiles of Drug Substances; Florey, K., Ed.; Academic Press, Inc.: San Diego, CA, 1989; Vol. 18, pp 221-288.

52. Boje, K. M.; Sak, M.; Fung, H-L. Complexation of Nifedipine with Substituted Phenolic Ligands. Pharm. Res. 1988, 5, 655-659.

53. Wohnsland, F.; Faller, B. High-Throughput Permeability pH Profile and High-Throughput Alkane/Water Log P with Artificial Membranes. J.Med.Chem. 2001,44, 923-930.

54. Rothwell, J. A.; Day, A. J.; Morgan, M. R. A. Experimental Determination of Octanol-Water Partition Coefficients of Quercetin and Related Flavonoids. J. Agric. Food Chem. 2005, 53, 4355-4360.

55. Ungell, A-L. In Vitro Absorption Studies and Their Relevance to Absorption from the GI Tract. Drug Dev. Ind. Pharm. 1997, 23, 879-892.

56. Navia, M. A.; Chaturvedi, P. R. Design Principles for Orally Bioavailable Drugs. Drug Discov. Today 1996, 1, 179-189.

57. Novalbos, J.; Abad-Santos, F.; Zapater, P.; Cano-Abad, M. F.; Moradiellos, J.; Sánchez-Garcia, P.; García, A. G. Effects of Dotarizine and Flunarizine on Chromaffin Cell Viability and Cytosolic Ca ${ }^{2+}$. Eur. J. Pharmacol. 1999, 366, 309-317.

58. Nifedipine Extended-Release Tablets Monograph (Test 2). In United States Pharmacopeia and National Formulary USP 32-NF 27; The United States Pharmacopeial Convention, Inc.: Rockville, 2009; p 3086.

59. Extended Release Oral Dosage Forms: Development, Evaluation, and Application of In Vitro/In Vivo Correlations; Guidance for Industry; U.S. Department of Health and Human Services, Food and Drug Administration, Center for Drug Evaluation and Research (CDER), U.S. Government Printing Office: Washington, DC, 1997.

60. Gray, V.; Dressman, J. B. Simulated Intestinal Fluid, TS-Change to $\mathrm{pH}$ 6.8. Pharm. Forum 1996, 22, 1943-1945.

61. Kavanagh, N.; Corrigan, O. I. Swelling and Erosion Properties of Hydroxypropylmethylcellulose (Hypromellose) Matrices - Influence of Agitation Rate and Dissolution Medium Composition. Int. J. Pharm. 2004, 279, 141-152.

62. Johnson, J. L.; Holinej, J.;Williams, M.D. Influence of lonic Strength on Matrix Integrity and Drug Release from Hydroxpropyl Cellulose Compacts. Int. J. Pharm. 1993, 90, 151-159.

63. Mitchell K.; Ford, J. L.; Armstrong, D. J.; Elliott, P. N. C.; Rostron, C.; Hogan, J. E. The influence of additives on the cloud point, disintegration and dissolution of hydroxypropylmethylcellulose gels and matrix tablets. Int. J. Pharm. 1990, 66 (1-3), 233-242.

64. Levina, M.; Vuong, H.; Rajabi-Siahboomi, A. R. The Influence of Hydro-Alcoholic Media on Hypromellose Matrix Systems. Drug Dev. Ind. Pharm. 2007, 33 (10), 1125-1134.

65. Fadda, H. M.; Mohamed, M. A.; Basit, A. W. Impairment of the In Vitro Drug Release Behaviour of Oral Modified Release Preparations in the Presence of Alcohol. Int. J. Pharm. 2008, 360, 171-176.

66. Traynor, M. J.; Brown, M. B.; Pannala, A.; Beck, P.; Martin, G.P. Influence of Alcohol on the Release of Tramadol from 24-h Controlled-Release Formulations During In Vitro Dissolution Experiments. Drug Dev. Ind. Pharm. 2008, 34 (8), 885-889.

67. Roberts, M.; Cespi, M.; Ford, J. L.; Dyas, A. M.; Downing, J.; Martini, L. G.; Crowley, P. J. Influence of Ethanol on Aspirin Release from Hypromellose Matrices. Int. J. Pharm. 2007, 332 (1-2), 31-37.

68. Calpena, A. C.; Lauroba, J.; Suriol, M.; Obach, R.; Domenech, J. Effect of d-Limonene on the Transdermal Permeation of Nifedipine and Domperidone. Int. J. Pharm. 1994, 103, 179-186.

69. Ku, M. S. Use of the Biopharmaceutical Classification System in Early Drug Development. AAPS J. 2008, 10, 208-212.

70. Galia, E.; Nicolaides, E.; Hörter, D.; Löbenberg, R.; Reppas, C.; Dressman, J. B. Evaluation of various dissolution media for predicting in vivo performance of Class I and II drugs. Pharm. Res. 1998, 15 (5), 698-705.

71. Pouton, C.W. Formulation of Poorly Water-Soluble Drugs for Oral Administration: Physicochemical and Physiological Issues and the Lipid Formulation Classification System. Eur. J. Pharm. Sci. 2006, 29, 278-287. 